\title{
Fabrication and application of carbohydrate microarray for analyzing human serum antibody-carbohydrate interaction
}

\author{
Gang-Liang Huang $\cdot$ Xin-Ya Mei $\cdot$ Han-Xing Zhang • \\ Peng-George Wang
}

Published online: 12 May 2007

(C) Springer-Verlag 2007

\section{Erratum to: Appl Microbiol Biotechnol}

DOI: 10.1007/s00253-006-0723-9

Since the online publication of this article, it has been brought to our attention that the authors are unable to reach an agreement regarding the article's authorship. The Editors and Springer would like to stress that the article does not meet the requirements of a scientific publication. Therefore, the Editors and Springer distance themselves from this article.

The online version of the original article can be found at http://dx.doi. org/10.1007/s00253-006-0723-9.

G.-L. Huang $(\bowtie) \cdot$ X.-Y. Mei $\cdot$ H.-X. Zhang $\cdot$ P.-G. Wang State Key Laboratory of Microbial Technology (SKLMT), Shandong University,

Jinan, Shandong 250100, People's Republic of China

e-mail: hg1226@126.com 